\title{
Quantification of Motor Function in Huntington Disease Patients Using Wearable Sensor Devices
}

\author{
Mark Forrest Gordon ${ }^{a} \quad$ Igor D. Grachev ${ }^{b}$ Itzik Mazehc Yonatan Dolan ${ }^{c}$ \\ Ralf Reilmannd, e Pippa S. Loupe ${ }^{f}$ Shai Fine ${ }^{g}$ Leehee Navon-Perry ${ }^{h}$ \\ Nicholas Gross $^{a} \quad$ Spyros Papapetropoulos ${ }^{i} \quad$ Juha-Matti Savolaj \\ Michael R. Hayden k, I

\begin{abstract}
a Specialty Clinical Development, Teva Pharmaceutical Industries Ltd., Frazer, PA, USA; ${ }^{b}$ Guide Pharmaceutical Consulting, LLC, Millstone Township, NJ, USA; ${ }^{c}$ Advanced Analytics Department, Intel, Petah Tikva, Israel; ${ }^{d}$ George Huntington Institute and Department of

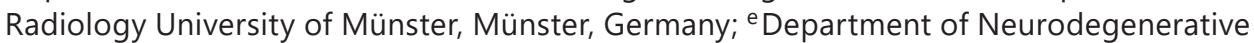

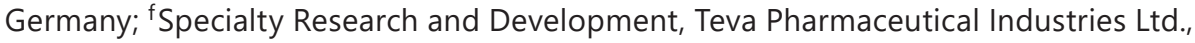
New Orleans, LA, USA; 9 Data Science Institute, Interdisciplinary Center, Herzliya, Israel; ${ }^{\mathrm{h}}$ Clinical Operations, Biometrics and Data Science, Netanya, Israel; 'Department of Neurology, Massachusetts General Hospital, Boston, MA, USA; ${ }^{j}$ Teva Pharmaceuticals International, Basel, Switzerland; kGlobal Research and Development, Teva Pharmaceutical Industries Ltd., Petah Tikva, Israel; 'Prilenia Therapeutics, Herzliya, Israel
\end{abstract} \\ Diseases and Hertie Institute for Clinical Brain Research, University of Tübingen, Tübingen,
}

\section{Keywords}

Activity monitors · Artificial intelligence $\cdot$ Digital devices $\cdot$ Digital health $\cdot$ Huntington disease $\cdot$ Motor symptoms $\cdot$ Chorea

\section{Abstract}

Previous studies have demonstrated the feasibility and promise of wearable sensors as objective measures of motor impairment in Parkinson disease and essential tremor. However, there are few published studies that have examined such an application in Huntington disease (HD). This report provides an evaluation of the potential to objectively quantify chorea in HD patients using wearable sensor data. Data were derived from a substudy of the phase 2 OpenPRIDE-HD study, where 17 patients were screened and 15 patients enrolled in the substudy and ultimately 10 patients provided sufficient wearable sensor data. The substudy was de- 
signed to provide high-resolution data to inform design of predictive algorithms for chorea quantification. During the entire course of the 6-month study, in addition to chorea ratings from 18 in-clinic assessments, 890 home assessments, and 1,388 responses to daily reminders, 33,000 h of high-resolution accelerometer data were captured continuously from wearable smartwatches and smartphones. Despite its limited sample size, our study demonstrates that arm chorea can be characterized using accelerometer data during static assessments. Nonetheless, the small sample size limits the generalizability of the model. The sensor-based model can quantify the chorea level with high correlation to the chorea severity reported by both clinicians and patients. In addition, our analysis shows that the chorea digital signature varies between patients. This work suggests that digital wearable sensors have the potential to support clinical development of medications in patients with movement disorders, such as chorea. However, additional data would be needed from a larger number of HD patients with a full range of chorea severity (none to severe) with and without intervention to validate this potentially predictive technology.

(C) 2019 The Author(s)

Published by S. Karger AG, Basel

\section{Introduction}

Huntington disease (HD) is a neurodegenerative disease caused by an abnormal cytosineadenine-guanine (CAG) triplet repeat expansion in $\mathrm{Htt}$, the gene that encodes the huntingtin protein. When the CAG repeat number is 36 or more, the mutated huntingtin (mHTT) becomes toxic, resulting in eventual neuronal dysfunction and death [1].

HD is characterized by progressive cognitive decline, psychiatric symptoms, and movement disorders including involuntary jerking or writhing chorea movements, incoordination, and bradykinesia. Chorea is common and usually occurs early in adult-onset HD. Assessment of chorea severity in the clinic can be done using the Unified Huntington's Disease Rating Scale (UHDRS) - Total Motor Score, which rates various motor signs including eye movements, speech, chorea, dystonia, rapidly alternating movements, bradykinesia, and gait [2]. Alternatively, motor disorders can be objectively and quantitatively assessed in the clinic using force transducer-based measures, as in the quantitative motor (Q-Motor) battery employed in the TRACK-HD study [3] and the PRIDE-HD study [4].

While movement-sensing devices such as Q-Motor [3] have been used successfully in the clinical setting, there are few studies examining their use in home settings with HD patients. The use of movement sensor devices can provide added value to the clinical assessment of movement disorders [5] by increasing the frequency of sampling and the ease of data collection, and allow for real-time objective monitoring of movement outside of the clinical setting [6]. Andrzejewski et al. [7] conducted a study of wearable sensors in the clinic and at home in patients with HD $(n=15)$ and controls $(n=5)$ to determine their feasibility and potential utility. The results of this study supported the feasibility of wearable sensors in both the clinic and home settings and demonstrated gait differences between participants with HD and healthy controls. The present study of digital devices was conducted as an observational substudy within the phase 2 Open-PRIDE-HD study, an assessment of the long-term efficacy and safety of pridopidine. Open-PRIDE-HD participants who provided specific informed consent were asked to wear sensory mobile devices to allow for the estimation of clinically relevant measures of HD. The objective of the digital substudy was to develop algorithms that could objectively quantify chorea by analyzing the data from the wearable sensors, to ultimately increase the clinical understanding of HD, and to provide insight into the daily variability of HD motor symptoms outside the clinical setting. 


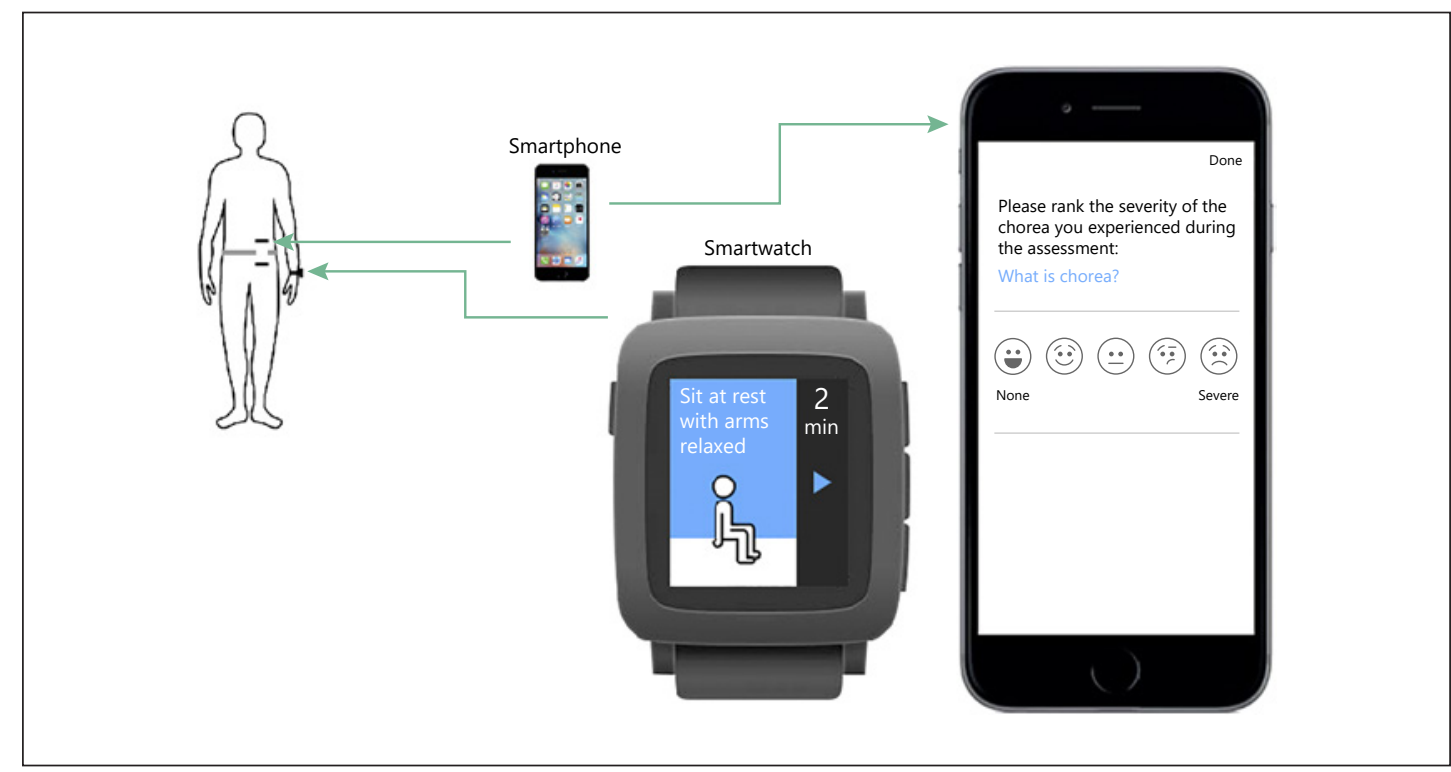

Fig. 1. Patients were asked to wear the smartwatch morning and night and to wear the smartphone near the smartwatch. The smartwatch displays one of the activities of the chorea assessment and the smartphone displays the chorea severity rating question.

\section{Methods}

\section{Participants}

Patients from eleven investigational Open-PRIDE-HD study sites were asked to participate in this device substudy. Eligible participants included male and female patients with HD (age $\geq 21$ years with a body weight of $\geq 50 \mathrm{~kg}$ ) who were enrolled in the Open-PRIDE-HD study and were treated with $45 \mathrm{mg}$ pridopidine twice daily (total daily dose $90 \mathrm{mg}$ ). Patients needed to demonstrate appropriate use of the digital wearable device and the smartphone application and compliance with the substudy requirements. A caregiver could assist in the use of the device and smartphone as needed.

\section{Procedure}

Patients were asked to report on their chorea symptoms for 6 months. For the in-clinic assessments, participants attended a clinic visit every 2 months and underwent an assessment of chorea severity using a seven-step structured test. The structured test was built into the mobile application and the tasks were performed in the following order: (1) timed up and go, (2) sit at rest with arms relaxed for $2 \mathrm{~min}$, (3) sit at rest with arms extended for $1 \mathrm{~min}$, (4) stand at rest for $30 \mathrm{~s}$, (5) walk in a straight line for $10 \mathrm{~m}$, (6) drink from a cup repetitively five times, and (7) pronation-supination (hand rotation) for $30 \mathrm{~s}$. To facilitate downstream analysis, a collective single score for all tasks (i.e., not seven individual scores), ranging between 0 (no chorea) to 4 (severe chorea), was provided by the clinician and likewise by the study participant as an electronically reported outcome in the mobile application.

At home, patients were asked to rate their chorea severity using two methods. One was a two-step assessment of chorea severity using a specifically designed smartphone application every other day and involved two activities: (1) sit at rest with arms relaxed for $2 \mathrm{~min}$, and (2) stand at rest for $30 \mathrm{~s}$. A single score was provided collectively for both activities by the study participant and the assessment times alternated between morning and evening. The second method consisted of a single report of their current chorea severity at a predefined 
time following a daily reminder from their smartphone. For every assessment, patients rated their chorea severity level on a scale from 0 (no chorea) to 4 (severe chorea).

In addition to chorea ratings, triaxial accelerometer data were collected from both the wearable smartwatch and the smartphone. The smartwatch was worn on the wrist of the chorea-dominant upper limb. If a chorea-dominant limb could not be determined, the smartwatch was worn on the nondominant limb. Patients were asked to wear the smartwatch continuously during daytime hours throughout the 6-month period. As illustrated in Figure 1, the smartphone was intended to be in proximity to the smartwatch (i.e., in the patient's waist pocket or holstered in the waist area). Study participants and caregivers received instructions on care and use of the smartwatch and smartphone as well as training on the chorea assessment applications. The training sessions were completed at the substudy screening visit by the site team. A check of compliance with the smartwatch and smartphone was performed during each in-clinic visit. Compliance with device use at home was captured through the mobile application and monitored by the sponsor. Study participants also managed their pridopidine medication regimen within the preinstalled application on the smartphone and received daily reminders, morning and evening, to report on their medication intake. Information on the methods used to assess the compliance of the patients in this substudy can be found in Cohen et al. [8].

The patient's smartwatch accelerometer data were collected continuously every day and the patient's smartphone accelerometer and gyroscope data were collected during chorea assessments. All accelerometer data were collected at a $50-\mathrm{Hz}$ sampling rate. Smartphone accelerometer inertial units were captured as $\mathrm{m} / \mathrm{s}^{2}$. Smartwatch accelerometer data, captured in units of milli-G, were converted to match the smartphone units by multiplying by 9.8065/1,000. Triaxial data were converted into signal magnitude

$$
\text { norm }=\sqrt{x^{2}+y^{2}+z^{2}}
$$

for downstream analysis. Mean subtraction was subsequently used to center the one-dimensional signal on the value zero. The first $2 \mathrm{~s}$ of each step in an assessment were omitted to reduce transition effects, such as pressing a button to initiate a task. For in-clinic assessments, the last $2 \mathrm{~s}$ of the "timed up and go," "walk," and "drink" tasks were omitted as well since the patients actively stopped these activities by pressing a button.

\section{Modeling Approach}

The modeling process was composed of several sequential steps. The input for the entire process was the accelerometer signal collected from the smartwatch, the smartphone, or both. The output was the chorea score and a prediction algorithm on whether or not chorea existed for every assessment.

The model was trained on the clinician ranking of chorea severity during the in-clinic assessments. Due to the small sample size and the lack of a sufficient number of samples for each chorea score, we could not train a model for predicting the score on a scale of 0-4. Thus, the clinicians' rankings were binarized. All samples with a label $\geq 1$ (i.e., 1, 2, 3, or 4), indicating the presence of chorea, were assigned a label of " 1 ." All samples with a label of 0 , indicating the absence of chorea, were assigned a label of " 0 ." The data were split into 5-s windows, which were the input for the model. There was a 50\% overlap (2.5 s) between subsequent windows. These values (i.e., the 5 -s sliding windows with $50 \%$ overlap) have been shown to be suitable for analyzing human motion based on wrist accelerometer data, both in published research [9] and in our previous internal research (unpublished data, Intel Corp.). Each 5-s window was considered a separate input. All windows in a given assessment contained the same label, as labels were provided per assessment. 
We extracted 167 features for each training example (5-s window). These features were derived both from the frequency domain, i.e., capturing frequencies that were prominent in the accelerometer data, revealing movement patterns that the patient had (e.g., Fourier and wavelet transforms) [10] and from the time domain, i.e., characteristics of the acceleration itself. Feature selection was performed to reduce the number of features to 50 using the Conditional Mutual Information Maximization algorithm [11], which iteratively selects features that add information to explain the target variable.

The chorea prediction model consisted of two stacked random forest classifiers. The inspiration for the stacked modeling approach originated from studies assessing Parkinson symptoms in home environments and studies assessing activity identification using bodymounted sensors [12-14]. After a thorough evaluation of numerous configurations, i.e., combinations of classifiers (random forest, XGBoost, and logistic regression), features, and parameters (such as window size mentioned above), we focused on a chorea prediction model consisting of two stacked random forest classifiers because it best optimized the sensitivity, the specificity, and the area under the receiver operating characteristic curve. The first random forest classifier used the 5-s window training examples as described above and the patient's score as a binary classification label in which 0 was used for no chorea and 1 indicating the presence of chorea. This output was then aggregated at the assessment level using ten summary statistics: mean, SD, and eight different percentiles. These values served as the feature vector to the second random forest classifier, which thus operated at the assessment level rather than the 5-s window level. The output of the second model was the chorea prediction score for the given assessment. The two-step approach was possible as a single label was provided for each assessment. The performance of the chorea prediction model was evaluated using fivefold cross-validation. Data were split into folds of assessments (i.e., intervals from a single assessment were all assigned to a single fold).

\section{Sensor Fusion}

We evaluated the model using either one sensor (smartwatch, smartphone) or both. As during the testing both devices were located in proximity on different body parts, we hypothesized that merging sensor data might improve chorea prediction performance. To do this, the data from each of the sensors were synchronized based on timestamp, making sure that the samples from the two sensors occurred within the same time window.

Sensor fusion was performed using the stacked model approach described above. Input feature vectors from each sensor were first processed by the initial random forest at the 5-s window level. Using data from the smartwatch and smartphone sensors, output from the initial models was aggregated at the assessment level. As in the single-sensor model, these aggregated values served as the feature vector (this time with double the number of features, 10 from each sensor) into the second random forest model, whose output served as the chorea prediction score for the assessment.

\section{Results}

\section{Patient Demographics and Disposition}

In total, 17 patients ( 9 males and 8 females) were screened for the substudy and 15 were enrolled (Table 1). For the screened patients, the mean (SD) for the age of the participants was 51 (12) years, the number of CAG repeats was 44 (3), the UHDRS - Total Motor Score was 37 (14), and the UHDRS - Total Functional Capacity score was 8 (2). Five patients were discontinued due to study closure of Open-PRIDE-HD and their data were omitted from the analyses. 
Table 1. Patient demographics and study disposition

\begin{tabular}{|c|c|c|c|c|c|c|c|c|c|c|}
\hline $\begin{array}{l}\mathrm{Pa}- \\
\text { tient } \\
\text { No. }\end{array}$ & Sex & $\begin{array}{l}\text { Age, } \\
\text { years }\end{array}$ & BMI & $\begin{array}{l}\text { CAG } \\
\text { repeats }\end{array}$ & $\begin{array}{l}\text { UHDRS- } \\
\text { TMS }\end{array}$ & $\begin{array}{l}\text { UHDRS- } \\
\text { TFC }\end{array}$ & $\begin{array}{l}\text { Burden of } \\
\text { disease }^{\mathrm{a}}\end{array}$ & $\begin{array}{l}\text { Days in } \\
\text { study, } n\end{array}$ & Study disposition & $\begin{array}{l}\text { Data } \\
\text { included in } \\
\text { analysis? }\end{array}$ \\
\hline 82 & M & 63 & 21.66 & 42 & 54 & 8 & 409.5 & 183 & completed 6 months & yes \\
\hline 94 & $\mathrm{~F}$ & 60 & 31.91 & 42 & 45 & 8 & 390.0 & 137 & completed 6 months & yes \\
\hline 63 & $\mathrm{~F}$ & 52 & 25.22 & 39 & 22 & 11 & 182.0 & 135 & completed 6 months & yes \\
\hline 51 & $\mathrm{~F}$ & 69 & 29.46 & 41 & 68 & 3 & 379.5 & 177 & completed 6 months & yes \\
\hline 74 & M & 44 & 24.39 & 47 & 29 & 7 & 506.0 & 201 & completed 6 months & yes \\
\hline 57 & M & 51 & 26.57 & 44 & 24 & 8 & 433.5 & 184 & completed 6 months & yes \\
\hline 8 & M & 74 & 29.90 & 40 & 29 & 8 & 333.0 & 173 & completed 6 months & yes \\
\hline 108 & $\mathrm{~F}$ & 46 & 32.65 & 42 & 23 & 13 & 299.0 & 190 & completed 6 months & yes \\
\hline 25 & M & 41 & 29.52 & 46 & 31 & 8 & 430.5 & 114 & completed 6 months & yes \\
\hline 45 & M & 48 & 25.67 & 44 & 30 & 11 & 408.0 & 173 & completed 6 months & yes \\
\hline 18 & $\mathrm{~F}$ & 60 & 34.48 & 42 & 58 & 5 & 390.0 & 1 & $\begin{array}{l}\text { discontinued due to } \\
\text { personal issues }\end{array}$ & no \\
\hline 79 & M & 33 & 22.23 & 53 & 49 & 6 & 577.5 & 3 & $\begin{array}{l}\text { discontinued due to } \\
\text { personal issues }\end{array}$ & no \\
\hline 68 & M & 37 & 29.80 & 46 & 22 & 8 & 388.5 & 28 & $\begin{array}{l}\text { discontinued due to trial } \\
\text { closure }\end{array}$ & no \\
\hline 36 & $\mathrm{~F}$ & 51 & 20.96 & 43 & 29 & 11 & 382.5 & 45 & $\begin{array}{l}\text { discontinued due to trial } \\
\text { closure }\end{array}$ & no \\
\hline 99 & $\mathrm{~F}$ & 54 & 22.66 & 44 & 44 & 10 & 459.0 & 37 & $\begin{array}{l}\text { discontinued due to trial } \\
\text { closure }\end{array}$ & no \\
\hline 11 & M & 43 & 26.82 & 45 & 38 & 9 & 408.5 & 30 & $\begin{array}{l}\text { discontinued due to trial } \\
\text { closure }\end{array}$ & no \\
\hline 102 & $\mathrm{~F}$ & 33 & 28.33 & 49 & 42 & 9 & 445.5 & 30 & $\begin{array}{l}\text { discontinued due to trial } \\
\text { closure }\end{array}$ & no \\
\hline
\end{tabular}

BMI, body mass index; CAG, cytosine-adenine-guanine; UHDRS-TFC, Unified Huntington's Disease Rating Scale - Total Functional Capacity; UHDRS-TMS, Unified Huntington's Disease Rating Scale - Total Motor Score. a The formula for burden of disease (age $\times$ [CAG - 35.5]) is from Frost et al. [15].

\section{In-Clinic and Home Assessments of Chorea Severity}

During the 13-month study, eighteen in-clinic assessments of chorea severity were performed. Eight patients had two in-clinic assessments and 2 patients had one assessment. As shown in Figure 2a, for the in-clinic assessments of chorea severity, the patient-ranked scores were distributed across the entire severity range: 5 patients ranked their chorea as 0 , 4 ranked it as 1 (slight), 4 ranked it as 2 (mild), 4 ranked it as 3 (moderate), and 1 patient ranked it as 4 (severe); however, clinicians ranked most patients $(n=12)$ as having chorea severity of 2 (mild). The correlation between the patient and physician reports was very low. There were four assessments in which the patients reported no chorea while the clinician reported chorea as mild (score $=2$ ); thus, clinicians generally tended to rate chorea levels higher than patients.

Patients completed 890 home assessments and provided 1,388 chorea reports following reminders in their home environment during the study period (Table 2). The median severity score in both cases was 2, although the distribution was more balanced during the home assessments (Fig. 2b). A very high correlation (Pearson $r=0.99$ ) was found between the patients' mean chorea severity reports at home assessment and the chorea reporting following the home reminder. The average chorea scores following home assessment and following patient self-assessment during clinic visits were also observed to be well correlated (Pearson $r=0.91$; Fig. 2c). 


\section{. - lㅣㄹal}

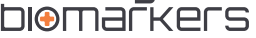

Digit Biomark 2019;3:103-115

DOI: $10.1159 / 000502136$ www.karger.com/dib

Gordon et al.: Quantification of Motor Function in HD Patients Using Wearable Sensor Devices

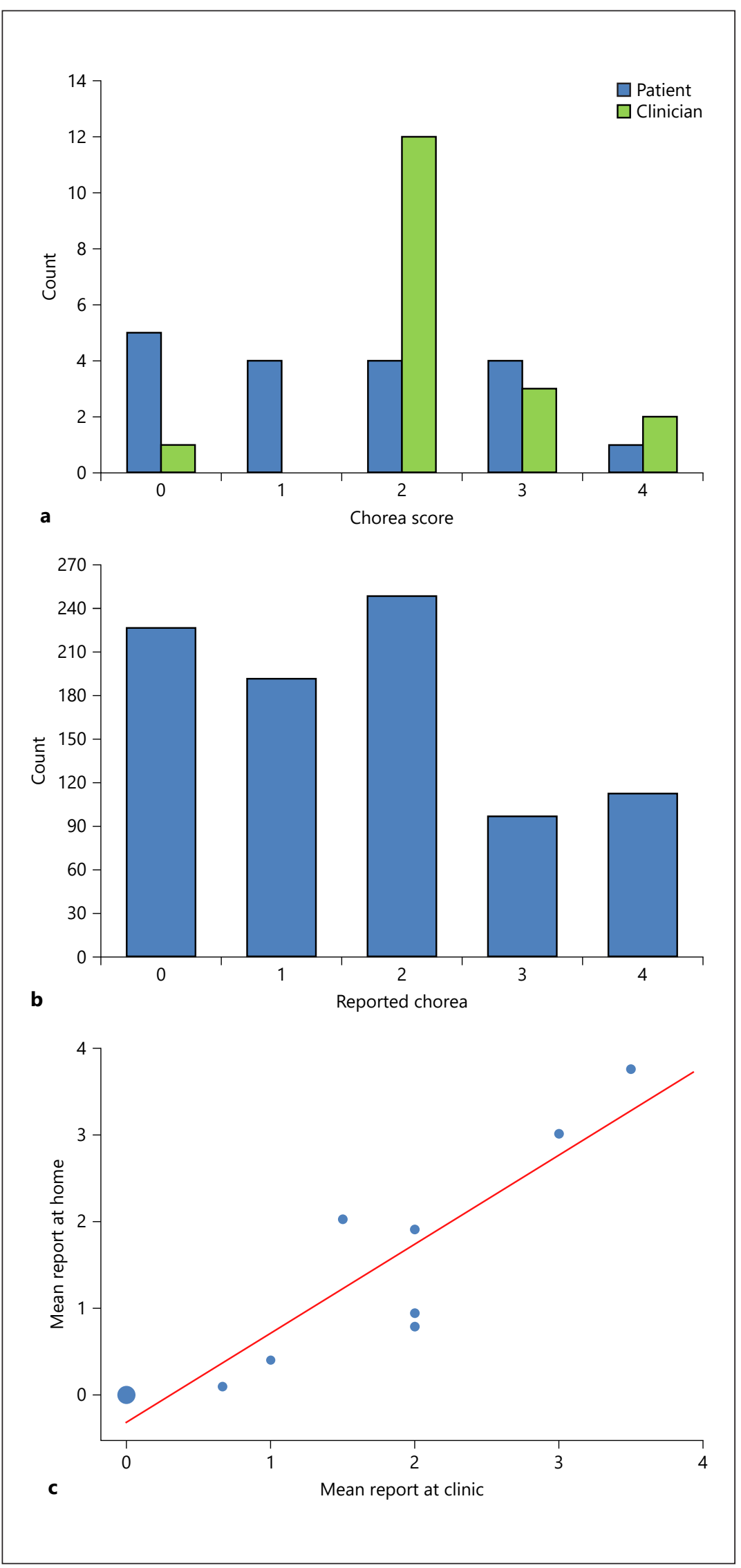

Fig. 2. In-clinic and home assessment of chorea severity. a Patients' and clinicians' chorea reports following clinic assessment. b Patients' chorea reports following home assessment. c Correlation between patients' chorea reports following home and clinic assessments. Circle size reflects the number of overlapping data points (i.e., the larger circle at 0,0 reflects the higher frequency of times patients ranked chorea severity level as 0 in both the home and clinic assessments). 
Gordon et al.: Quantification of Motor Function in HD Patients Using Wearable Sensor Devices

Table 2. In-clinic and home assessments and digital data collected during the study

\begin{tabular}{rlclll}
\hline $\begin{array}{l}\text { Patient } \\
\text { No. }\end{array}$ & $\begin{array}{l}\text { In-clinic } \\
\text { assess- } \\
\text { ments, } n\end{array}$ & $\begin{array}{l}\text { Actual/expected } \\
\text { home assessments, } \\
n(\%)^{\mathrm{a}}\end{array}$ & $\begin{array}{l}\text { Severity reports } \\
\text { following daily } \\
\text { reminders, } n\end{array}$ & $\begin{array}{l}\text { Smartwatch } \\
\text { accelerometer } \\
\text { data, } \mathrm{h}\end{array}$ & $\begin{array}{l}\text { Smartwatch } \\
\text { accelerometer } \\
\text { data points, } \times 10^{6}\end{array}$ \\
\hline 82 & 2 & $80 / 91(88 \%)$ & 157 & 4,032 & 710 \\
94 & 2 & $36 / 68(53 \%)$ & 118 & 2,511 & 432 \\
63 & 2 & $60 / 67(89 \%)$ & 131 & 2,489 & 423 \\
51 & 2 & $137 / 88(156 \%)$ & 113 & 3,916 & 658 \\
74 & 1 & $153 / 100(153 \%)$ & 276 & 3,569 & 596 \\
57 & 2 & $110 / 96(115 \%)$ & 334 & 3,530 & 797 \\
8 & 1 & $70 / 86(81 \%)$ & 69 & 3,373 & 580 \\
108 & 2 & $72 / 95(76 \%)$ & 180 & 1,839 & 276 \\
25 & 2 & $24 / 57(42 \%)$ & 1 & 3,918 & 676 \\
45 & 2 & $148 / 86(172 \%)$ & 9 & 33,589 & 5,653 \\
\hline Total & 18 & $890 / 834(106 \%)$ & 1,388 & 2505 \\
\hline
\end{tabular}

${ }^{\text {a }}$ Per protocol patients were expected to complete a home assessment every other day.

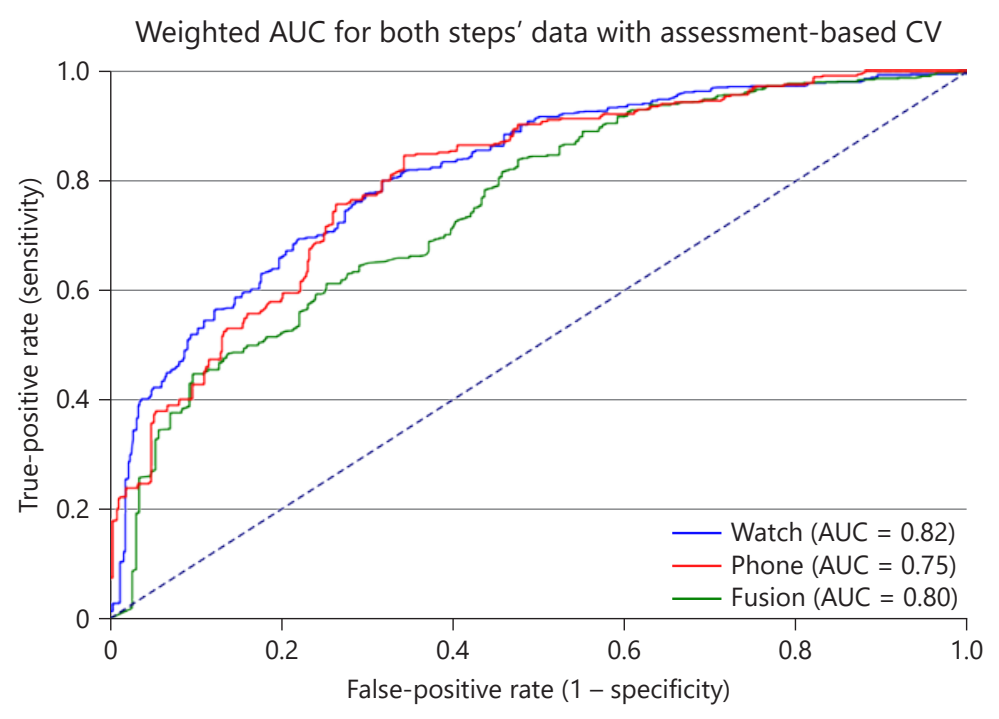

Fig. 3. Receiver operating characteristic curve of models in three scenarios: smartwatch accelerometer data, smartphone accelerometer data, and fusion of both. In order to give every patient an equal weight during the evaluation phase, the area under the curve (AUC) scores were weighted accordingly, giving every patient an inverse weight with respect to the frequency in the population. "Frequency in the population" refers to the number of assessments a specific patient performed divided by the entire collection of assessments from all patients. $\mathrm{CV}$, coefficient of variation.

\section{Model Performance}

The model was evaluated using the receiver operating characteristic curve. The receiver operating characteristic curve was created for our selected model configuration (i.e., for data originating from both steps in home assessments) in three different scenarios as shown in Figure 3: (1) only smartwatch accelerometer data were used (solid blue line), (2) only smart- 
Gordon et al.: Quantification of Motor Function in HD Patients Using Wearable Sensor Devices

Table 3. Confusion matrix of the model

\begin{tabular}{llll}
\hline & Chorea $=0$ predicted & Chorea $=1$ predicted & Total predicted \\
\hline Chorea $=0$ true & $N_{00}=164$ & $N_{01}=63$ & $N_{0 \mathrm{x}}=227$ \\
Chorea $=1$ true & $N_{10}=100$ & $N_{11}=551$ & $N_{1 \mathrm{x}}=651$ \\
\hline Total true & $N_{\mathrm{x} 0}=264$ & $N_{\mathrm{x} 1}=614$ & $N=878$ \\
\hline
\end{tabular}

phone accelerometer data were used (solid green line), and (3) fusion of smartwatch and smartphone accelerometer data (solid red line). The fusion-based model did not appear to outperform the single-sensor models and therefore no statistical testing was performed.

Table 3 shows the confusion matrix that was generated following selected (sensorfusion) model prediction, based on a threshold of 0.7. The corresponding scores for this configuration are: sensitivity $=85 \%(551 / 651)$, specificity $=72 \%(164 / 227)$, positive predictive value (precision) $=90 \%(551 / 614)$, accuracy $=81 \%(164+551) /(878)$, and negative predictive value $=62 \%(164 / 264)$.

\section{Association between Model Prediction and Labels}

As expected, there was a correlation between the model chorea score and the patientreported chorea score for the same assessment (Fig. 4a). There was also an overall trend of an increase in the model predicting chorea as the clinician's report of chorea severity increased (Fig. 4b). The model appeared to be more accurate with clinician-reported chorea levels of 1 and 3 and less accurate with a clinician-reported chorea level of 2 . No clinician had patients with an average chorea severity level of 0 or 4 .

Upon examination of each individual patient as shown in Figure 4c, it becomes apparent that the model provided chorea scores in alignment with the patient-reported chorea scores for patients who reported low chorea scores (e.g., patients 8, 63, and 108 who reported a chorea level of 0 ) and those who reported high chorea scores (e.g., patients 82 and 51 who reported chorea levels of 3 and 4, respectively). For patients 25 and 45, the model's prediction of chorea levels was less aligned, but in these cases there was relatively good alignment between the model's chorea score and the clinician's chorea assessment.

\section{Discussion}

Although the speed of disease progression in HD varies, patients exhibit a predictable clinical profile and inexorably develop disability, eventually leading to death usually 15-20 years after diagnosis. Chorea and other motor symptoms experienced by HD patients have a significant impact on their lives, and having an objective measure of the clinical progression of symptoms can aid in evaluating the effectiveness of potential treatments [16]. The digital health substudy within the Open-PRIDE-HD study served as an initial platform to assess the feasibility, value, and features of this wearable technology combined with artificial intelligence in monitoring the disease and measuring abnormal movement.

A limitation of the study is the low number of patients enrolled, which did not allow development of a more generalizable model and more substantial conclusions. Another limitation was the closure of the Open-PRIDE-HD study, which led to variation in the patients' participation time. This resulted in limited data collection from 5 of the 17 enrolled patients and the subsequent removal of their data from the analyses. Despite these limitations, our study suggests that chorea can be characterized using accelerometer data during static 
Gordon et al.: Quantification of Motor Function in HD Patients Using Wearable Sensor Devices

Fig. 4. The model's prediction of chorea. a The model's prediction of chorea categorized by the patients reported symptom score of chorea. $N$ is the number of reports. $\mathbf{b}$ The model's prediction of chorea categorized by the clinicians' reported level of chorea severity score. $N$ is the number of reports. c Scatter plots of association between the model's chorea prediction and chorea values reported by patients (left) and clinicians (right). Left panel: the patients' average chorea report at home ( $x$ axis) is plotted against the model's average chorea probability score ( $y$ axis). Right panel: the clinicians' average chorea report in the clinic ( $x$ axis) is plotted against the model's average chorea probability score ( $y$ axis). Every point represents a single patient; the number depicts the patient ID.
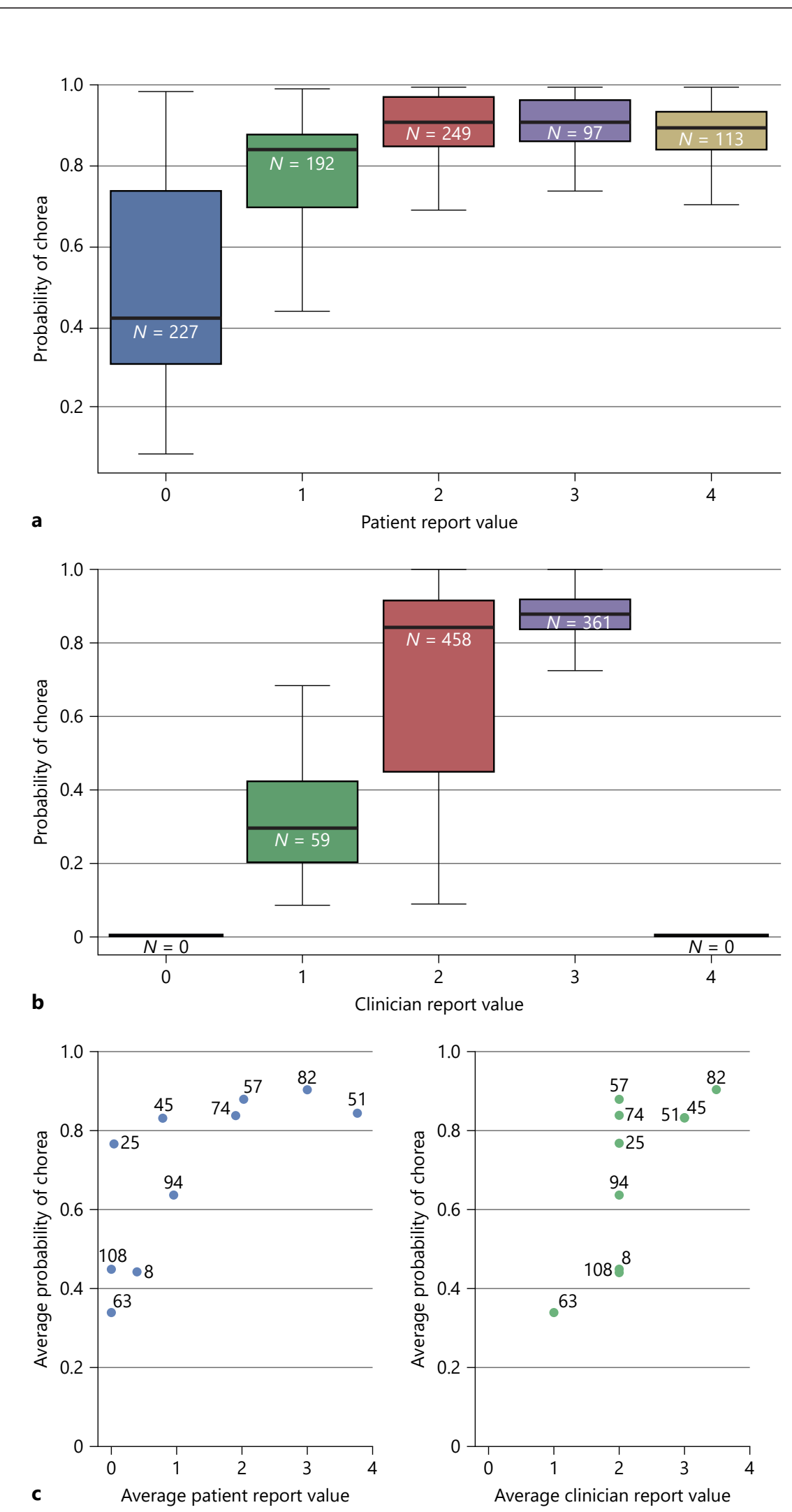
assessments. Furthermore, the results show the association between the chorea level quantified by the sensor-based model and the chorea scores reported by the clinicians and the patients. This association appears to be stronger for the clinician-reported than for the patient-reported chorea scores.

Interestingly, our analysis suggests that chorea's digital signature varies between patients and has personalized characteristics, e.g., some patients rated their chorea as nonexistent (0) or with lower severity than was predicted by the model and inconsistent with the in-clinic assessment. These results are not surprising, as previous studies have shown that selfawareness of chorea is impaired in HD patients [17]. Using wearable sensory devices may increase patient engagement in health monitoring, increase their self-awareness, and prevent variability in assessments. In this study, patients and caretakers were trained on how to use the devices, and this may have increased self-awareness of chorea and compliance with this study. Compliance is further described in Cohen et al. [8]. The use of devices to assess chorea in patients with HD was further supported by the results of the phase 2 parent study PRIDE-HD. In PRIDE-HD, utilization of the Q-Motor task, choreomotography, provided objective, non-rater-biased information in a randomized clinical trial that was not detectable in clinical scales $[3,18]$.

In summary, our results suggest that wearable digital sensors may have the potential to support clinical development of medications in patients with chorea. However, additional studies involving a larger number of patients with a full range of chorea severity (none to severe), with and without intervention, are needed to validate this approach. The ability to monitor the patient in the home setting may have many advantages, and the information collected may be of great potential value for treating clinicians and for those developing better therapies for movement disorders. Symptom monitoring through the use of a wearable device may also improve patient adherence, assist in treatment decisions, limit medical costs, and reduce pressure on healthcare resources. The data collected from these wearable devices may facilitate the development of medications for HD and other neurodegenerative diseases.

\section{Acknowledgment}

We gratefully acknowledge the dedication of the participants and caregivers of the digital substudy and the investigators and staff at the participating sites.

\section{Statement of Ethics}

Both the Open-PRIDE-HD study and the digital device substudy were conducted in accordance with good clinical practice and US FDA guidelines. The Open-PRIDE-HD study is registered at clinicaltrials.gov as NCT02494778 and in the EudraCT database as 2015-000904-24. All protocols, including the digital substudy protocol, were approved by the institutional review boards for each site, and patients provided written specific informed consent before participating in the substudy.

\section{Disclosure Statement}

M.F. Gordon, I.D. Grachev, P.S. Loupe, S. Fine, L. Navon-Perry, N. Gross, S. Papapetropoulos, J.-M. Savola, and M.R. Hayden are or were Teva Pharmaceutical Industries employees during the course of this study. I. Mazeh and Y. Dolan are employees of Intel. R. Reilmann is 
founding director and owner of the George Huntington Institute, a private research institute focused on clinical and preclinical research in HD, and QuantiMedis, a clinical research organization providing Q-Motor services in clinical trials and research. He serves as an elected member of the steering committees of the European Huntington's Disease Network and the Huntington Study Group, co-chair of the Task Force on Huntington's Disease, and member of the Task Force on Technology of the International Parkinson and Movement Disorder Society. He has provided consulting services, advisory board functions, clinical trial services, Q-Motor analyses, or lectures for Actelion Pharmaceuticals, Amarin Neuroscience, AOP Orphan Pharmaceuticals, the Cure Huntington Disease Initiative Foundation, Desitin, Hoffmann-La Roche, Ionis Pharmaceuticals, Ipsen, Lundbeck, Link Medicine, Meda Pharma, Medivation, Mitoconix, Neurocrine Biosciences, Neurosearch, Novartis, Omeros, Pfizer, Prana Biotechnology, Prilenia Therapeutics, Raptor Pharmaceuticals, Siena Biotech, Temmler Pharma, Teva Pharmaceuticals, uniQure, Vaccinex, Wave Life Sciences, and Wyeth Pharmaceuticals. He has received grant support from the Bundesministerium für Bildung und Forschung, the Cure Huntington Disease Initiative Foundation, the Deutsche Forschungsgemeinschaft, the Deutsches Zentrum fur Neurodegeneration und Entzündung, the European Union Seventh Framework Program (EU-FP7), the European Huntington's Disease Network, the High Q Foundation, and the National Science Foundation.

\section{Funding Sources}

Teva Pharmaceutical Industries Ltd. sponsored the Open-PRIDE-HD study and the digital substudy described in this report.

\section{Author Contributions}

M.F. Gordon provided project administration, data interpretation, visualization, and drafting and critical revision of the manuscript. I.D. Grachev was the study physician responsible for digital substudy design, protocol development, medical monitoring, data collection and interpretation, and provided review of the manuscript. I. Mazeh and Y. Dolan were involved in conceptualization, data collection and interpretation, project administration, validation, and provided critical revision of the manuscript. R. Reilmann was the principal investigator of the PRIDE-HD study and provided conceptualization, supervision, resources, and review of the manuscript. P.S. Loupe provided data interpretation, visualization, wrote the initial draft, and managed manuscript development. S. Fine was the co-lead for the digital substudy, responsible for study design and execution, monitoring, data collection and interpretation, and critical review of the manuscript. L. Navon-Perry was responsible for digital substudy protocol drafting and finalization and provided data interpretation and critical review of the manuscript. N. Gross provided data analysis and interpretation of the results and review of the manuscript. S. Papapetropoulos, J.-M. Savola, and M.R. Hayden contributed to study design and execution, project management, resources, funding acquisition, and review of the manuscript. 


\begin{tabular}{|c|c|}
\hline \multicolumn{2}{|l|}{ ait Biomark $2010 \cdot 2 \cdot 103$} \\
\hline DOI: 10.1159/000502136 & $\begin{array}{l}\text { (c) } 2019 \text { The Author(s). Published by S. Karger AG, Basel } \\
\text { www.karger.com/dib }\end{array}$ \\
\hline
\end{tabular}

Gordon et al.: Quantification of Motor Function in HD Patients Using Wearable Sensor Devices

\section{References}

1 Bates GP, Dorsey R, Gusella JF, Hayden MR, Kay C, Leavitt BR, et al. Huntington disease. Nat Rev Dis Primers. 2015 Apr;1(1):15005.

2 Huntington Study Group. Unified Huntington's Disease Rating Scale: reliability and consistency. Mov Disord. 1996 Mar;11(2):136-42.

3 Reilmann R, Schubert R. Motor outcome measures in Huntington disease clinical trials. Handb Clin Neurol. 2017;144:209-25.

4 Reilmann R, McGarry A, Grachev ID, Savola JM, Borowsky B, Eyal E, et al.; European Huntington's Disease Network; Huntington Study Group investigators. Safety and efficacy of pridopidine in patients with Huntington's disease (PRIDE-HD): a phase 2, randomised, placebo-controlled, multicentre, dose-ranging study. Lancet Neurol. 2019 Feb;18(2):165-76.

5 Espay AJ, Bonato P, Nahab FB, Maetzler W, Dean JM, Klucken J, et al.; Movement Disorders Society Task Force on Technology. Technology in Parkinson's disease: challenges and opportunities. Mov Disord. 2016 Sep; 31(9):1272-82.

6 Hirsch IB, Martinez J, Dorsey ER, Finken G, Fleming A, Gropp C, et al. Incorporating Site-less Clinical Trials Into Drug Development: A Framework for Action [Internet]. Clin Ther. 2017 May;39(5):1064-76.

7 Andrzejewski KL, Dowling AV, Stamler D, Felong TJ, Harris DA, Wong C, et al. Wearable Sensors in Huntington Disease: A Pilot Study. J Huntingtons Dis. 2016 Jun;5(2):199-206.

8 Cohen S, Waks Z, Elm JJ, Gordon MF, Grachev ID, Navon-Perry L, et al. Characterizing patient compliance over six months in remote digital trials of Parkinson's and Huntington disease. BMC Med Inform Decis Mak. 2018 Dec;18(1):138.

9 Janidarmian M, Roshan Fekr A, Radecka K, Zilic Z. A comprehensive analysis on wearable acceleration sensors in human activity recognition. Sensors (Basel). 2017 Mar;17(3):529.

10 Preece SJ, Goulermas JY, Kenney LP, Howard D. A comparison of feature extraction methods for the classification of dynamic activities from accelerometer data. IEEE Trans Biomed Eng. 2009 Mar;56(3):871-9.

11 Fleuret F. Fast binary feature selection with conditional mutual information. J Mach Learn Res. 2004;5:153155.

12 Das S, Amoedo B, De la Torre F, Hodgins J. Detecting Parkinson's symptoms in uncontrolled home environments: a multiple instance learning approach. Conf Proc IEEE Eng Med Biol Soc. 2012;2012:3688-91.

13 Banos O, Galvez JM, Damas M, Pomares H, Rojas I. Window size impact in human activity recognition. Sensors (Basel). 2014 Apr;14(4):6474-99.

14 Preece SJ, Goulermas JY, Kenney LP, Howard D, Meijer K, Crompton R. Activity identification using bodymounted sensors - a review of classification techniques. Physiol Meas. 2009 Apr;30(4):R1-33.

15 Frost C, Mulick A, Scahill RI, Owen G, Aylward E, Leavitt BR, et al.; TRACK-HD Investigators. Design optimization for clinical trials in early-stage manifest Huntington's disease. Mov Disord. 2017 Nov;32(11):1610-9.

16 Kieburtz K, Reilmann R, Olanow CW. Huntington's disease: current and future therapeutic prospects. Mov Disord. 2018 Jul;33(7):1033-41.

17 Sitek EJ, Sołtan W, Wieczorek D, Schinwelski M, Robowski P, Reilmann R, et al. Self-awareness of motor dysfunction in patients with Huntington's disease in comparison to Parkinson's disease and cervical dystonia. J Int Neuropsychol Soc. 2011 Sep;17(5):788-95.

18 Reilmann R, Rouzade-Dominguez ML, Saft C, Süssmuth SD, Priller J, Rosser A, et al. A randomized, placebocontrolled trial of AFQ056 for the treatment of chorea in Huntington's disease. Mov Disord. 2015 Mar;30(3): 427-31. 